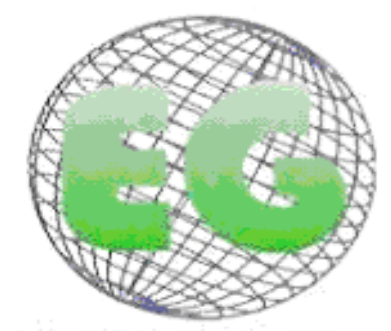

ISSN 1695-6141

$\mathrm{N}^{\circ} 23$
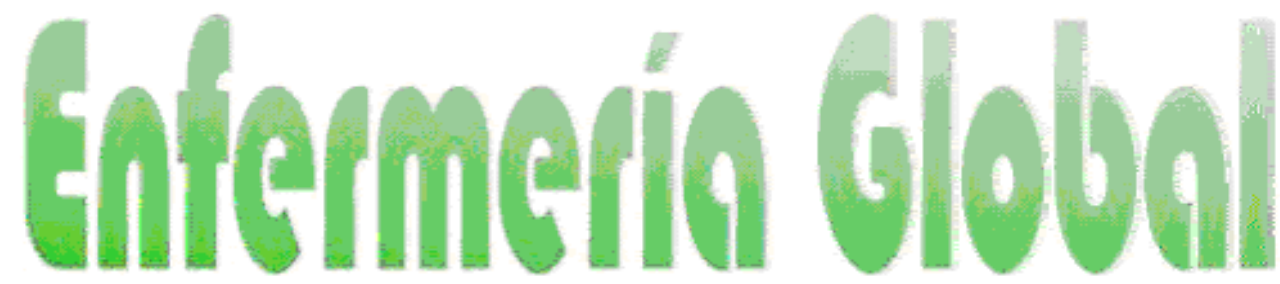

Revista electrónica trimestral de Enfermería

www.um.es/egloball

DOCENCIA - INVESTIGACIÓN

\title{
Instrumentos para la enseñanza del proceso enfermero en la práctica clínica docente con enfoque de autocuidado utilizando Nanda-Nic-Noc
}

Tools for teaching nurses in clinical teaching with the focus on Nanda-Nic-Noc

*González Sara, SH., "*Moreno Pérez, NE.

*Estudiante de Maestría en Ciencias de la Enfermería. ** Maestra en Administración. Docente. División de
Ciencias de la Salud e Ingenierías del Campus Celaya - Salvatierra. Universidad de Gto. México.

Palabras clave: Proceso enfermero; Taxonomías NANDA, NIC NOC.

Keywords: Nursing process; NANDA, NIC, NOC, Taxonomies

\section{RESUMEN}

La herramienta metodológica en la práctica científica de enfermería es el Proceso Enfermero, el cual nos permite emplear como método de resolución de problemas el pensamiento crítico, el uso de esta metodología ha venido a dar autonomía a la profesión y su uso es determinante en la práctica clínica y la docencia.

Objetivo: Desarrollar habilidades en la implementación desarrollo y evaluación de un diseño de enfermería con enfoque de Autocuidado, en la utilización de las taxonomías NANDA, NIC NOC. Metodología. Se analizó la Teoría del Déficit de Autocuidado y las etapas del Proceso Enfermero, se construyeron dos instrumentos: 1) Razonamiento diagnóstico: incluye las etapas de valoración y diagnóstico, y 2) Plan de cuidados: comprende las etapas de planificación, ejecución y evaluación del proceso enfermero.

Resultados. Los instrumentos se aplicaron durante la práctica clínica de las áreas de Materno infantil, Adulto Mayor y Enfermería Comunitaria, participaron estudiantes y docentes, previo curso taller que incluyó: Teoría general del Autocuidado, Taxonomías NANDA, NIC, NOC y estructura y llenado de los instrumentos, los casos clínicos se presentaron en un foro al final de la práctica clínica. Conclusiones los instrumentos se consideraron recursos que facilitan la enseñanza del PE en los estudiantes de enfermería que están incorporándose a las prácticas clínicas.

\section{ABSTRACT}

The metgodological tool for the scientific practice of nursing is the Nursing Process, which enables critical thinking in solving problems. The use of this methodology has bestowed autonomy on the profession and its use is important in clinical practice and teaching.

Aim: To develop skills in implanting, developing and assessing a nursing design with the focus on self-care using NANDA NIC NOC. 
Methodology: The Self-Care Defecit Theory and the stages of the Nursing Process were analysed and two tools were constructed: 1) Diagnostic Reasoning, which includes valoration and diagnosis; 2) Care Plan: the planning, execution and assessment of the Nursing Process.

Results: The tools were applied to clinical practice in the areas of Mother-child, Older Adults and Community Nursing. Students and teachers participated after following a workshop course on General Theory of Self-Care, NANDA, NIC, NOC Taxonomies and structure and content of the tools, Clinical cases were presented in a final forum on clinical practicea.

Conclusions: The tools were considered to be resources that help in teaching the NP to students of nursing beginning to work in clinical practices.

\section{INTRODUCCIÓN}

La educación en enfermería en los últimos años ha tenido avances importantes, producto de la preocupación constante por mejorar la calidad, por la innovación de tecnologías que permiten estar al día en los conocimientos y los procesos relacionados al cuidado y a las demandas de salud de los últimos años. Sin embargo, a pesar de estos avances, uno de los retos sigue siendo el abordaje de los problemas de enfermería de forma metódica y con fundamento científico, a través de la aplicación del Proceso Enfermero (PE) en la práctica enfermera. Sabemos que su diseño e implementación no es fácil y precisamente este hecho ha determinado su aceptación o rechazo, por lo tanto, el desarrollo de instrumentos que faciliten su aplicación cobra gran importancia sobre todo en la enseñanza pedagógica de enfermería.

La aplicación del PE desde lo pedagógico permite iniciar a los estudiantes en la aplicación de la metodología científica y a la aproximación de las teorías en enfermería aplicables a nuestra población (práctica científica) que sean útiles para resolver y atender las demandas de cuidados de nuestros clientes o pacientes. ${ }^{(1)}$

El PE es el "Método sistemático y organizado de administrar cuidados de enfermería individualizados, que se centra en la identificación y tratamiento de las respuestas del paciente a las alteraciones de salud reales o potenciales", es la herramienta metodológica que facilita la aplicación de la resolución científica de problemas a los cuidados, permitiendo una visión holística de las personas, y consta de cinco etapas: Valoración, diagnóstico, planificación, ejecución y evaluación. La aplicación de la teoría, así mismo, es útil para el razonamiento y la toma de decisiones en la práctica, permite organizar y entender aquello que pasa alrededor del cuidado, posibilita el juicio clínico y la reflexión filosófica al planificar los cuidados, proponer intervenciones de enfermería, predecir y explicar resultados del paciente y evaluar la eficacia de los cuidados, explica la identidad y el objetivo de la práctica enfermera.

En la Enfermería práctica una de la teorías más populares y utilizadas en América Latina es el Modelo de Dorothea E. Orem, con su Teoría General del Déficit de Autocuidado (TGDA); este es uno de los modelos más estudiados y aplicado en distintos ámbitos sanitarios y en diversas edades de la población con éxito. ${ }^{(2)}$

La TGDA permite identificar claramente el por qué y el cómo las personas cuidan de sí mismas, cómo la enfermera puede ayudar y explica las relaciones que deben existir para que se produzca el cuidado enfermero, por lo tanto, en este modelo se enmarca lo que entendemos por cuidados de enfermería e integra a su vez los conceptos de autocuidado, autocuidado terapéutico, agencia de autocuidado y agencia de cuidado dependiente como conceptos claves. ${ }^{(3)}$ 
El docente en enfermería es responsable en gran medida de los conocimientos que adquiere el estudiante frente al reto de brindar cuidados holísticos con base científica de manera organizada, la mejor manera para lograrlo es estimular la actualización, mediante el uso de la bibliografía más novedosa en la enseñanza de los estudiantes, lo cual brindará motivación para su autopreparación en actividades docentes y técnicas participativas. Debido a lo anterior, es imprescindible para el diseño de instrumentos que se incluyan los esquemas nuevos de apoyo al desarrollo del cuidado, que sean claros y sencillos, como lo son las clasificaciones NIC y NOC.

El desarrollo de esta estructura proporciona un marco actual y sencillo para la elaboración de un diseño de enfermería (plan de cuidados) para la enseñanza en los entornos educativos; de esta manera se buscó construir unos instrumentos que permitieran al estudiante de enfermería de pregrado desarrollar habilidades en la implementación, desarrollo y evaluación de un diseño de enfermería, así como que integrara y desarrollara habilidad en el uso de la Metodología de Proceso aplicando el enfoque Teórico de Dorothea E. Orem y usando en la planificación de los cuidados la metodología multiaxial: NANDA, NIC, NOC.

\section{METODOLOGÍA}

Para la construcción de estos instrumentos primeramente fue necesario realizar un análisis de la Teoría General del Déficit de Autocuidado en la versión más reciente de la obra de Orem. ${ }^{(4)}$ Se revisó y comprendió cada uno de los conceptos y elementos que comprenden la teoría así como cada una de las cinco etapas del PE.

Validación. Se presentó la propuesta al personal docente de una facultad de enfermería en un taller de Proceso Enfermero con Enfoque de Orem, posteriormente se implementó un taller con los estudiantes sobre las experiencias educativas de Enfermería del Adulto, Enfermería Infantil y Enfermería Comunitaria para el adiestramiento y para la aplicación en la práctica clínica de la metodología NANDA, NIC-NOC, se tomó el acuerdo de utilizar los formatos de valoración con enfoque de autocuidado ya establecidos en la facultad, los resultados en el uso de estos instrumentos se evaluaron en un foro de presentación de casos clínicos al final del periodo de prácticas clínicas, se demostró la factibilidad de la aplicación de la teoría estudiada y de esta metodología con la utilización de los instrumentos de valoración vigentes en la facultad.

La recolección de los datos se hizo a partir de los instrumentos de valoración conocidos por el estudiante basados en la identificación de los factores condicionantes Básicos, Requisitos de Autocuidado Universales, Requisitos de Autocuidado de desarrollo y Requisitos de Autocuidado de Desviación de la salud.

Los instrumentos se organizaron en dos partes, la primera de ellas muestra el proceso del razonamiento diagnóstico y el pensamiento crítico, se basa en el análisis de los datos de la valoración; implica la investigación y el acúmulo de factores que inciden sobre la capacidad de autocuidado, las demandas de autocuidado de la persona y la naturaleza de la relación entre ambas (Factores condicionantes básicos). De igual forma se deben especificar los Requisitos de Autocuidado Universales, los cuales representan las acciones de la persona a partir de sus condiciones tanto internas como externas, en total son ocho: mantenimientos de un aporte suficiente de agua, aire, suficiente alimento, provisión de cuidados asociados con la eliminación, mantenimiento del equilibrio entre la actividad y el reposo, mantenimiento del equilibrio para la vida, el funcionamiento y el bienestar humano, promoción del funcionamiento y desarrollo humano dentro de los grupos sociales. 
Los Requisitos de Autocuidado de Desarrollo son las expresiones más especificas de la persona (fisiológica, cronológica de los procesos vitales o acontecimiento de la vida) y Requisitos de autocuidado de Desviación de la salud son los que están presentes cuando existe una enfermedad o están en proceso de diagnóstico y/o tratamiento, esencialmente este instrumento permite determinar la demanda y establecer el déficit de autocuidado (real o de riesgo) para esta etapa. Aunque Orem da su propia propuesta de diagnósticos, en la aplicación de este instrumento se decidió mantener el lenguaje de la taxonomía NANDA planteado un Diagnóstico Enfermero utilizando la metodología PES (Problema, etiología, signos y síntomas) ${ }^{(5)}$

Factores Condicionantes Básicos:

Nombre:

Médico: No. De Cama:

INSTRUMENTO I

RAZONAMIENTO DIAGNÓSTICO

Escolaridad Edo. Civil: Edad: Género: Fecha: Diagnóstico Servicio: Religión: Peso: Talla: Ocupación: Tipo de seguridad social: Tipo de Familia

Con cuántas personas vive: Rol en su familia: De quién depende económicamente

Servicios con que cuenta su:

Domicilio:

Colonia o comunidad:

\begin{tabular}{|c|c|c|c|c|}
\hline $\begin{array}{c}\text { Requisitos } \\
\text { Universales }\end{array}$ & $\begin{array}{c}\text { Requisitos } \\
\text { de }\end{array}$ & $\begin{array}{c}\text { Requisitos } \\
\text { de } \\
\text { Desarrollo } \\
\text { Desviación } \\
\text { de la Salud }\end{array}$ & $\begin{array}{c}\text { Demanda de } \\
\text { Autocuidado }\end{array}$ & $\begin{array}{c}\text { Diagnóstico de } \\
\text { Enfermería }\end{array}$ \\
\hline & & & & \\
& & & & \\
& & & &
\end{tabular}

Nombre del Estudiante: 
Tutor Clínico:

El segundo instrumento comprende las etapas de planificación, ejecución y evaluación del $\mathrm{PE}$, se conduce al estudiante de enfermería para que desarrolle acciones prescriptivas haciendo posible las operaciones de tratamiento o reguladoras partiendo del planteamiento del diagnóstico. Este segundo instrumento incluye que se participe en operaciones interpersonales, sociales y operaciones tecnológico- profesionales. ${ }^{(6)}$ Para el desarrollo de las etapas de planeación, ejecución y evaluación se utiliza la clasificación de resultados de enfermería (NOC) y la clasificación de Intervenciones de Enfermería (NIC). Una vez que se determinan las acciones de enfermería para el beneficio de otros y los objetivos en salud, se establece la manera y el contexto en los que la enfermera y la persona interactúan señalando los elementos de rol de la persona, el sistema de enfermería y la función de enfermería; la estructura de este segundo instrumento llamado Plan de cuidados se divide en los siguientes apartados:

1. Apartado Déficit de Autocuidado: Determinar la capacidad de autocuidado y las demandas de autocuidado presentes y futuras de la persona (diagnóstico de enfermería Taxonomía NANDA).

2. Apartado Resultado: Determinar el estado, conducta o percepción, variables de un individuo, familia o comunidad medido a lo largo de un continuo en respuesta a intervención(es) de enfermería. ${ }^{(7)}$

3. Apartado Indicadores: Estado o conducta o percepción de un individuo familia o comunidad más concreto que sirve para medir un resultado.

4. Apartado Escala de Likert: Medida de cinco puntos que cuantifica un resultado del individuo o un estado del indicador sobre un continuo desde menos a más deseable y proporciona una puntuación en un momento dado.

5. Apartado agencia de Enfermería: Establecer el rol del individuo, familia o comunidad, rol de la enfermera y rol de otras personas implicadas en el cuidado en la satisfacción de acciones de autocuidado.

6. Apartado sistema de Enfermería: Establecer el rol del individuo, familia o comunidad, rol de la enfermera y rol de las otras personas implicadas en el cuidado en la regulación de acciones de autocuidado.

7. Apartado intervenciones: Todo tratamiento basado en el conocimiento y juicio clínico, que realiza un profesional de enfermería para favorecer el resultado esperado del individuo, familia o comunidad. ${ }^{(8)}$

8. Apartado actividades de enfermería: Acciones que realiza la enfermería para llevar a cabo una intervención y que ayudan al individuo, familia o comunidad a avanzar hacia el resultado deseado. ${ }^{(9)}$

9. Apartado fundamentación: Revisión científica de las actividades

10. Apartado métodos de ayuda: Establecer los cuidados directos de enfermería que se ofrecerán al individuo, familia o comunidad.

11. Apartado evaluación: Determinar los intervalos de tiempo deseados en función de la característica o concepto del resultado. Especificar la puntuación en relación al resultado. 


\section{INSTRUMENTO II. PLAN DE CUIDADOS}

Nombre:

Diagnóstico Médico:

Género:

Fecha:

Cama:

\section{Déficit de Autocuidado}

\begin{tabular}{|c|c|c|c|c|c|}
\hline 2. Resultado & 5. Agencia & 6. Sistema & \begin{tabular}{|c|}
7. \\
Intervencio \\
nes
\end{tabular} & 8. Actividades & 9. Fundamentación \\
\hline \multicolumn{3}{|c|}{ 3. Indicadores 4. Escala de Likert } & & & \\
\hline & 2 & 45 & & & \\
\hline \multicolumn{6}{|l|}{ Total Likert } \\
\hline \multicolumn{3}{|c|}{ 11. Evaluación } & $\begin{array}{l}\text { 10. Métodos } \\
\text { de Ayuda }\end{array}$ & & \\
\hline Fecha/Turno & & & & & \\
\hline $\begin{array}{l}\text { Puntuación } \\
\text { Likert }\end{array}$ & & & & & \\
\hline
\end{tabular}

\section{RESULTADOS}

Los instrumentos fueron aplicados durante el desarrollo de las prácticas clínicas correspondientes a las Experiencias Educativas del área Materno Infantil, Enfermería del Adulto y Enfermería Comunitaria las cuales tuvieron una duración de seis meses. Se consideró importante que los estudiantes, previo a la práctica clínica, recibieran una capacitación con el objetivo de favorecer el aprendizaje y desarrollaran la habilidad en tres ejes principales: Teoría de Dorothea E. Orem, Proceso Enfermero y estructura y llenado de los instrumentos.

Al final de la práctica clínica los resultados de la implementación de los instrumentos se presentaron en un foro científico en donde los estudiantes presentaron procesos enfermeros elaborados por ellos mismos y supervisados y dirigidos por su tutor clínico ante expertos docentes, personal asistencial de las principales Instituciones de Salud de la localidad y autoridades universitarias, así mismo, se les pidió que además emitieran comentarios con el objeto de conocer dificultades y fortalezas en la aplicación y uso de los instrumentos con 
los conceptos de la TGDA. La mayoría de los participantes refirieron pocas dificultades con el llenado de los instrumentos y manifestaron satisfacción en la organización de los conceptos y distribución de la información en ambos instrumentos.

\section{CONCLUSIONES}

El uso de los instrumentos facilitó el desarrollo de cada una de las etapas del PE, los participantes consideraron que con la implementación de los mismos se integran los conceptos del modelo teórico a las etapas del PE contribuyendo de esta manera al fortalecimiento de la ciencia de enfermería; con los comentarios dejaron en claro que los instrumentos resultaron innovadores en la integración de los elementos de la TGDA y de la metodología NANDA, NIC y NOC, en consecuencia, se consideraron recursos que facilitan la enseñanza del PE en los estudiantes que están incorporándose a las prácticas clínicas.

\section{REFERENCIAS BIBLIOGRÁFICAS}

1. Vega O.M, González ED. Teoría del Déficit de Autocuidado: Interpretación desde los Elementos Conceptuales. Revista Ciencia y Cuidado Volumen 4 N0 4 ISSN-17949831

2. López D, A. Perspectiva Internacional en el Uso de la Teoría General de Orem. Rev. Investigación y Educación en Enfermería Vol. XXIV N. 2 [consultado mayo 2010] disponible en: http://www.scielo.org.co/pdf/iee/v24n2/v24n2a10.pdf

3. Marriner-Tomey. Modelos y Teorías de Enfermería. 5ta. Ed. Mosby/Doyma. 2007 España.

4. Orem E, D. Modelo de Orem Conceptos de Enfermería en la Práctica. Ed. MASSON SALVAT. 1993, Barcelona España.

5. NANDA. Diagnósticos Enfermeros, definiciones y clasificación 2007-2008. Ed. Elsevier. 2008. Madrid

6. Cavanagh S,J. Modelo de Orem, Aplicación práctica. Ediciones Científicas y Técnicas. Masson-Salvat. 1993. España.

7. Moorhead Sue et al, Clasificación de Resultados de Enfermería (NOC), $3^{\text {a }}$ edición, editorial Elsevier-Mosby, 2005. Madrid España,

8. Ostiguín M,R. Velázquez HSM. Teoría General del Déficit de Autocuidado. Guía básica ilustrada. Manual Moderno. 2001 México

9. Johnson Marion et al, Diagnósticos Enfermeros, Resultados e Intervenciones, NIC. ed. Harcourt-Mosby, 2002, Barcelona España, 